\title{
A Novel Transmission Electron Imaging Technique for Observation of Whole Cells
}

Yusuke Ominami ${ }^{1}$, Masato Nakajima ${ }^{2}$, Tatsuo Ushiki ${ }^{2}$, Sukehiro Ito ${ }^{1}$

${ }^{1}$. Hitachi High-Technologies Corporation, 882, Ichige, Hitachinka-Shi, Ibaraki-ken, 312-8504, Japan

2 . Niigata University Graduate School of Medical and Dental Sciences, 1-757 Asahimachi-dori, Chuo-ku, Niigata-city, Niigata-ken, 951-9510, Japan

The ultrastructure of biological tissues and cells has been studied mainly by transmission electron microscopy (TEM) and scanning transmission electron microscopy (STEM). For this purpose, samples usually need to be embedded in plastic resin and cut into ultrathin sections for mounting them onto mesh grids. Meanwhile, correlative light and electron microscopy (CLEM) for observing same samples using light and electron microscope have been also recently reported by some investigators. By light microscopy, samples are normally placed on a transparent plate (i.e., a glass slide or a dish), which makes it difficult to investigate the internal structure using TEM and STEM. Thus, by CLEM, the observed sample has to be further sliced into ultrathin sections and placed on mesh grids after observation with a light microscope. To overcome this technical complexity, we propose, in this study, a novel and simple technique for transmission electron imaging by scanning electron microscopy (SEM) for observing samples on plate. The configuration of the conventional TEM and the proposed technique are shown in Fig. 1(a) and (b). In this technique, samples are placed on a transparent and flat plate in advance which is made of a scintillator material that emits photons by electron irradiations (Fig. 1 (b)). Electrons are backscattered by internal structures of the samples with higher density or penetrated into structures with lower density, thus generating the varying number of photons from the plate. As a result, the contrast of obtained image is produced by transmitted electrons (TEs).

In order to evaluate the utility of the proposed technique, muntjac cells (MM2T) were cultivated on the scintillator plates in microplate wells (Fig. 1(c)). After cultivation, the samples were fixed in 1\% glutaraldehyde and stained with $1 \% \mathrm{OsO}$, dehydrated in a ETOH series and critical point-dried. The SEM used in this study was a Hitachi SU3500 tungsten emission SEM with a photo detector. Images of cultured cells on the plate are shown in Fig. 2. This technique enables the wide-filed observation of whole cells at lower magnification because all cells are directly placed not on mesh grid but on a plate for detecting TEs. This TE image with internal cell information can be clearly compared with the image obtained by secondary electrons (SEs), which has information on the cell surface structure (Fig. 2(a) 
and (b)). High magnification images clearly show structures inside cells (Fig. 2(c) and (d)). Stereo-pair images can be obtained by tilting an incident electron beam at $\pm 5^{\circ}$ (Fig. 2(e)). In conclusion, the proposed technique is very useful for obtaining TE images of whole cells with a conventional SEM without the use of any destructive sample-thinning process.

(a)

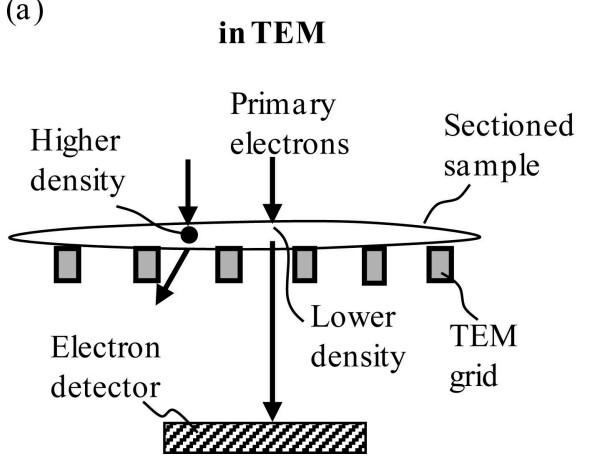

(b)

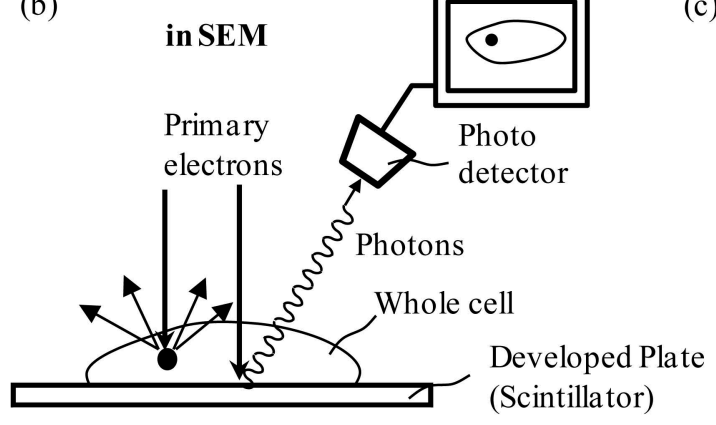

(c)

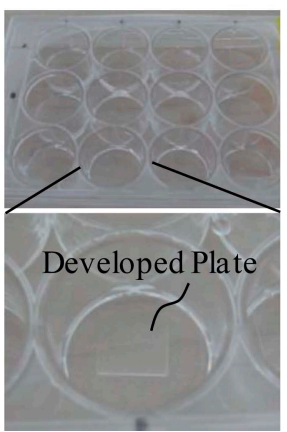

Fig. 1 Configuration of the conventional TEM imaging (a) and the proposed TE imaging (b) methods. (c)(d), the developed STEM plate on a well of the microplate.

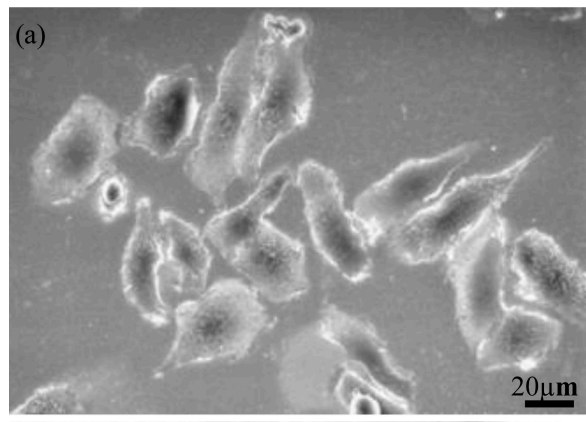

(c)
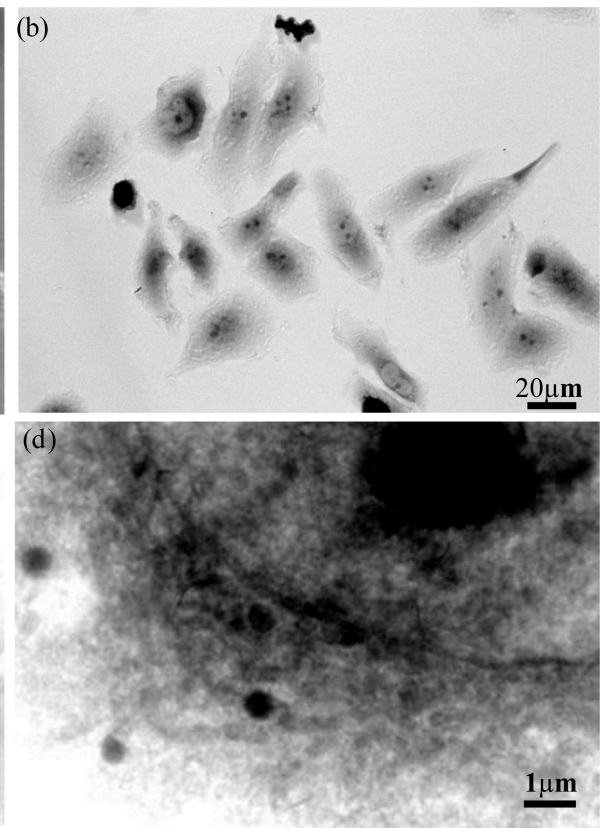

(e)

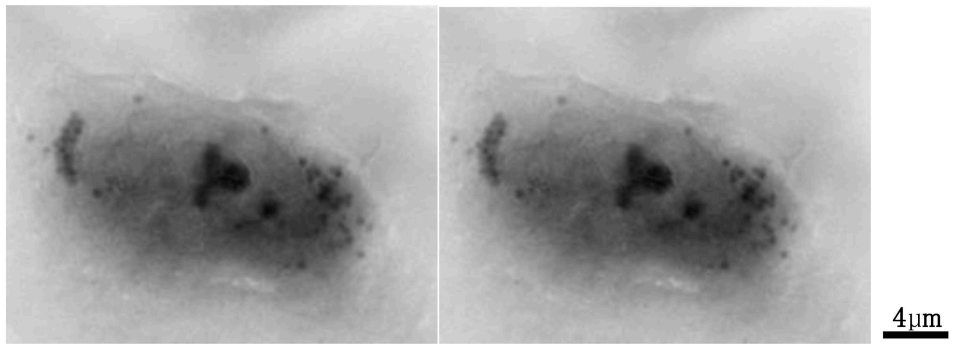

Fig. 2 (a) SE image and (b) TE image of cells on the plate. (c)(d) TE images at x5, 000 (c) and x10,000 (d) magnifications (e) Stereo-pair TE images of whole cells. 